\title{
Collision cascade effects near an edge dislocation dipole in alpha-Fe: induced dislocation mobility and enhanced defect clustering
}

\author{
S. Heredia-Avalos ${ }^{\mathrm{a}, *}$, C. D. Denton ${ }^{\mathrm{b}}$, J. C. Moreno-Marín ${ }^{\mathrm{a}}$, E. Martinez ${ }^{\mathrm{c}}$, M. J. \\ Caturlab \\ ${ }^{a}$ Dept. de Física, Enginyeria de Sistemes $i$ Teoria del Senyal, Universitat d'Alacant, \\ Apartat 99, E-03690 Alacant, Spain \\ ${ }^{b}$ Dept. de Física Aplicada, Universitat d'Alacant, Apartat 99, E-03690 Alacant, Spain \\ ${ }^{c}$ Theoretical Division, T-1, Los Alamos National Laboratory Los Alamos, 87545, NM, USA
}

\begin{abstract}
Collision cascades near a $1 / 2\langle 111\rangle\{110\}$ edge dipole in alpha-iron have been studied using molecular dynamics simulations for a recoil energy of $20 \mathrm{keV}$ and two temperatures, $20 \mathrm{~K}$ and $300 \mathrm{~K}$. These simulations show that the collision cascade induces the migration of the dislocations through glide along its slip plane. The motion of the dislocations starts at the peak of the collision cascade and expands a time scale much longer than the cascade duration, until restoring the equilibrium distance of the dipole, regardless of the damage produced by the cascade. At the initial stages, kinks are formed at the dislocation that enhance glide. When defects reach the dislocations, jogs are produced. We show that the initial dislocation motion is triggered by the shock wave of the collision cascade. The cascade morphology is also strongly influenced by the presence of the dislocations, having an elongated form at the peak of the displacement, which demonstrates the strong interaction of the dislocations with the cascade even at the early stages. Finally, we show that larger vacancy clusters are formed in the presence of dislocations compared to isolated cascades and that these clusters are larger for $300 \mathrm{~K}$ compared to $20 \mathrm{~K}$.
\end{abstract}

Keywords: molecular dynamics, metals, dislocations, collision cascades,

\footnotetext{
${ }^{*}$ Corresponding author

Email address: sheredia@ua.es (S. Heredia-Avalos)
}

Preprint submitted to Journal of Nuclear Materials

September 29, 2020 
radiation damage

\section{Introduction}

The mechanical behavior of metals is governed by the characteristics and mobility of their dislocations. In the presence of defects produced by irradiation, this behavior can be altered modifying the plastic response of the material.

5 Effects such as hardening or loss of plasticity are common in metals exposed to irradiation [1, 2. The development of radiation resistant materials for fusion applications can benefit from a fundamental understanding of dislocation-defect interactions [3].

Molecular dynamics with empirical potentials has been an important tool to provide atomic level information about these phenomena. Most of the calculations performed consider the interaction of a single dislocation with a defect under an applied stress [4, 5, 6. The interaction of collision cascades with preexisting dislocations has been studied only recently [7, 8, 9, 10, 11, with most of the calculations performed in f.c.c. crystals [7, 8, 11 and, to a lesser extent, 15 in b.c.c. metals [9, 10. These simulations have shown how damage produced by the cascade can induce significant changes in the dislocation structure leading to dislocation climb or cross-slip [11] and, in some cases, glide [9]. Calculations in b.c.c. iron next to a single $1 / 2\langle 111\rangle\{110\}$ edge dislocation have been performed at low recoil energies $(5 \mathrm{keV})$ [10] showing a dependence of the cascade damage on the distance between the dislocation and the initial recoil, with enhanced vacancy clustering at certain distances and reduced defect production for both vacancies and self-interstitials at closer distances from the dislocation.

In this work, we have studied the effect of collision cascades produced by 20 $\mathrm{keV}$ recoils on a $1 / 2\langle 111\rangle\{110\}$ edge dipole and two different temperatures, 20

${ }_{25} \mathrm{~K}$ and $300 \mathrm{~K}$. After the two dislocations reach their equilibrium distance, an initial energetic recoil is started in the center between the two dislocations. These calculations show that the collision cascade induces glide of the two dislocations, something also observed previously in simulations done in tungsten [9, although 
not studied in detail. The importance of interstitials on dislocation climb has

30 sured the displacement produced by the two dislocations and study the effect of the defects produced by the cascade on this motion. Even more interesting is the strong influence of the dislocations on the morphology of the collision cascade, even at the very early stages. Finally, we have analyzed the damage compare the results with those in a pristine lattice, without the presence of the dislocations.

\section{Methodology}

We have used in our simulations a b.c.c. Fe crystal constituted by 1.7 million $z$ axes correspond to the $[2 \overline{1} \overline{1}],[01 \overline{1}]$ and [111] directions respectively. The

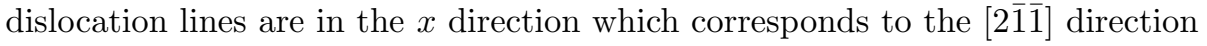
of the b.c.c. crystal and the $y$ axis is perpendicular to the glide plane of the dislocation. Our cell has dimensions of approximately $283 \AA \times 280 \AA \times 245 \AA$

45 in the $x, y$, and $z$ directions respectively. The simulations are performed using the Large-scale Atomic/Molecular Massively Parallel Simulator (LAMMPS) 13, 14, with the Fe potential developed by Ackland et al. 15. A Langevin [16] heat bath is located at the borders of the simulation cell, except in the two planes perpendicular to the dislocation lines. Periodic boundary conditions are used throughout the 3 directions. First of all, we have let the system evolve for 200 ps at a constant temperature $(300 \mathrm{~K}$ or $20 \mathrm{~K})$ and both dislocations move in the $z$ direction until reaching a new equilibrium position as shown in Figure 11(a). Figure 1 (b) shows the position of the dislocations as a function of time during the thermalization up to $200 \mathrm{ps}$. The dislocations reach an equilibrium position at least within this time scale, at $300 \mathrm{~K}$ a slight motion could be observed.

After the dipole has reached the equilibrium position, a collision cascade 


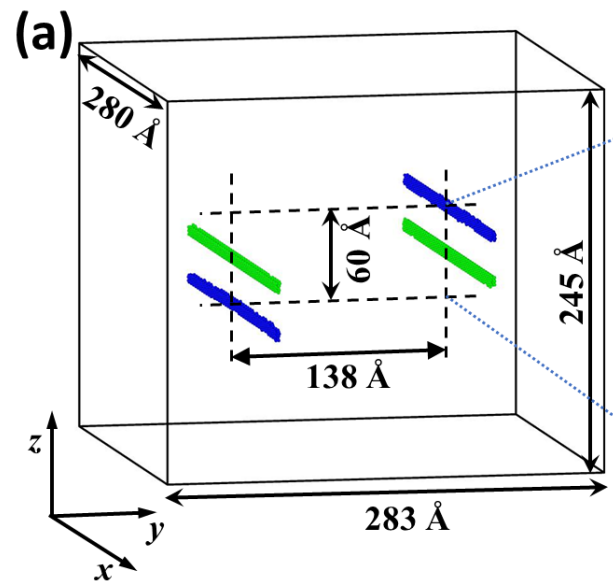

(b)

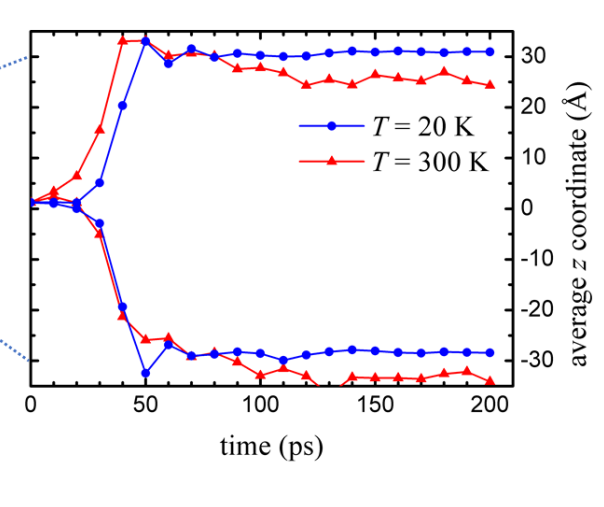

Figure 1: (colour online) (a) Simulation configuration used in this work. The initial position of the atoms belonging to the dislocations can be observed in green (light colour) while their equilibrium position after a thermalization at $20 \mathrm{~K}$ during $100 \mathrm{ps}$ is plotted in blue (dark colour). (b) Average $z$ coordinate of the two dislocations as a function of time during thermalization at $20 \mathrm{~K}$ and $300 \mathrm{~K}$.

is started by giving $20 \mathrm{keV}$ of energy to a central atom in slightly different directions close to the $-z$ direction, that is, perpendicular to the dislocation line and away from it. Note that the distance between the initial recoil and either of the two dislocations along the $y$ direction is $\approx 70 \AA$. We let the collision evolve for up to $60 \mathrm{ps}$ and analyze how the collision cascade affects the position of the dislocations and how the dislocations affect the creation of point defects of the cascade.

In order to analyze our results, we have used the open visualization tool OVITO [17, which also provides analysis software for atomistic simulation data. The movement of the dislocations was followed using a Common Neighbour Analysis (CNA) [18, whereas the positions of point defects created by the cascade were obtained using a Wigner-Seitz cell method, comparing the damaged cell with the cell before the cascade. The Dislocation Extraction Analysis (DXA) [19, implemented in OVITO, is used to identify the changes in the dislocations induced by the collision cascade. 


\section{Results and Discussion}

\subsection{Cascade induced dislocation glide}

The first noticeable effect observed in these calculations is that the two dislocations in the dipole are displaced from their equilibrium position when the collision cascade is initiated. In order to study the motion of both dislocations after the $20 \mathrm{keV}$ collision cascade in the crystal thermalized at $T=20 \mathrm{~K}$ (or $300 \mathrm{~K}$ ), we have identified the atoms close to the dislocation core with CNA [17, 18, choosing only those atoms that do not have a b.c.c. environment. This allows us to discard the atoms of the cell that do not belong to the dislocations. The motion of the dislocations is mostly in the $z$ direction, remaining fixed the $y$ coordinate of both dislocations, except when there is climb induced by the collision cascade, as explained below. Therefore, we can quantify the position of the dislocations by averaging the $z$ coordinate of the atoms around the dislocation core. The initial configuration of the dislocations before the cascade is shown in figure 1(a), identified using the method just described. Green atoms correspond to the position of the dislocations before relaxation and blue are the positions after they reach equilibrium. From the average position of the dislocation we obtain that one of the dislocations is initially located at $z \approx 30$ $\AA$ and the second one at $z \approx-30 \AA$ after equilibrium.

In figure 2(a) we show the average $z$ coordinate of both dislocations as a function of time for 10 different cascades at a temperature of $20 \mathrm{~K}$. They correspond to slightly different variations of the velocity direction of the primary knock-on atom (PKA) in order to obtain significant statistical fluctuations of the collision cascade, but always close to the $-z$ direction. We observe that in all cases both dislocations move in the $z$ direction. However, the average $z$ distance between dislocations can be increased or reduced after the starting of the collision cascade, as shown in figure 2(b). Eventually they reach an equilibrium distance which is approximately the same as the initial one. Note that, even though the equilibrium distance is constant, there is a slight drift of the two dislocations in some cases (see black lines). Such motion is not observed in the 


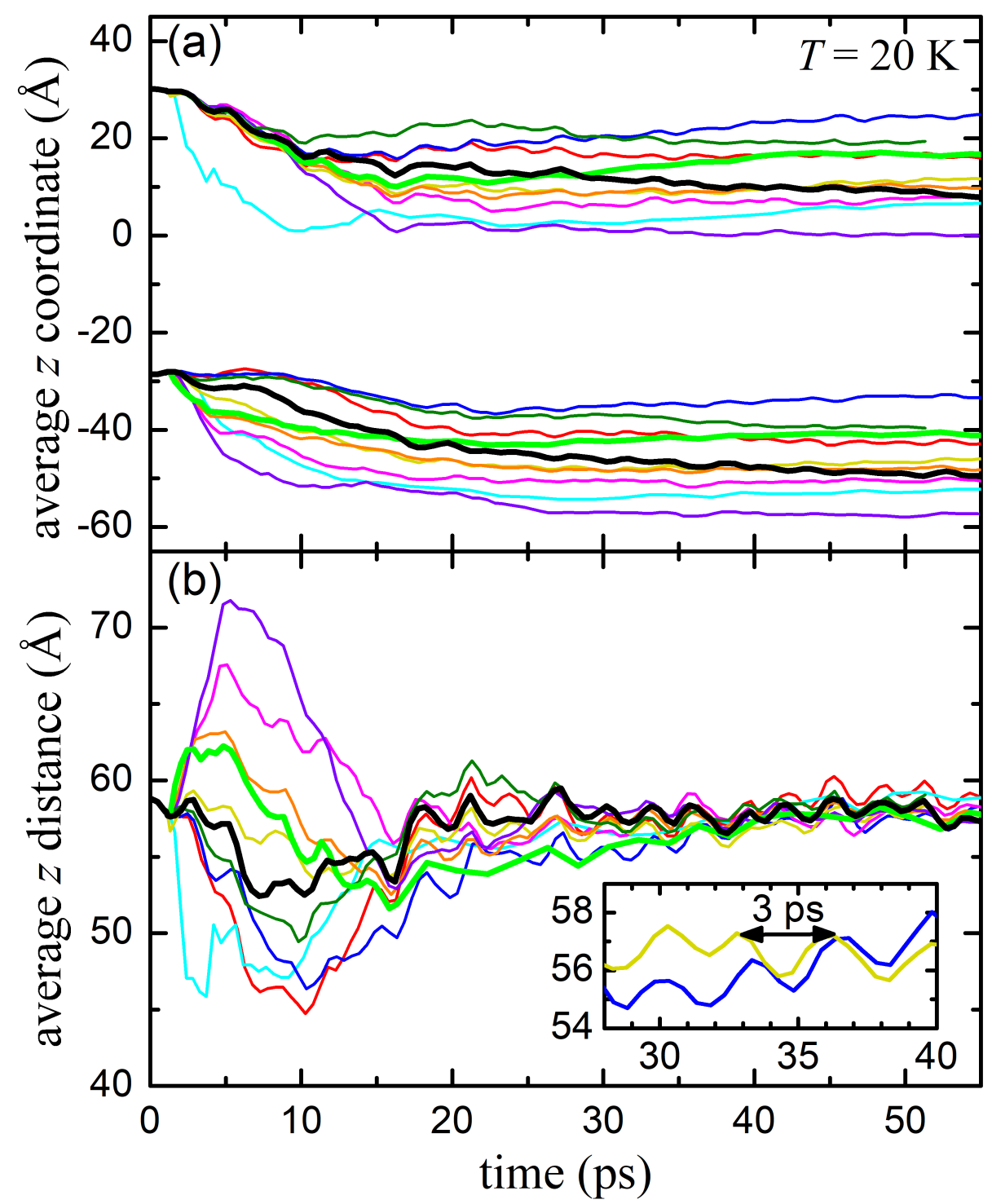

Figure 2: (colour online) Evolution of (a) the average $z$ coordinate of each dislocation and (b) the $z$ distance between dislocations, after a $20 \mathrm{keV}$ cascade close to the center of the simulation box and on the $-x$ direction in a bcc Fe with two edge dislocations at $T=20 \mathrm{~K}$. 
calculations at constant temperature before the cascade for the same time scale (see figure 1(b)).

Analogously, in figure 3(a) we observe the motion of the dislocations after a $20 \mathrm{keV}$ cascade for a temperature of $300 \mathrm{~K}$. Unlike for $T=20 \mathrm{~K}$, now in all cases both dislocations tend to move closer together after the collision, and after $10 \mathrm{ps}$ they tend to separate to reach again an equilibrium distance, similar to the initial one. This is better observed in figure 3 (b). The distance between dislocations is initially reduced, but after 10 ps it increases again, reaching an equilibrium distance similar to the initial one. Again oscillations in the distance between dislocations with a period of 3 ps can be observed. Also, at this temperature, the drift of the two dislocations after reaching the equilibrium distance is more clear than at $20 \mathrm{~K}$.

It is interesting to note the oscillations in the distance between dislocations, that appear with a period of around 3 ps (see insets in figures 2(b) and 3 (b)). Since the size of the simulation box is $245 \AA$ along the $z$ direction, the speed of this wave is $\approx 8176 \mathrm{~m} / \mathrm{s}$, which is above the speed of sound in iron. Therefore, this oscillation could be related to the shock wave induced by the collision cascade that does not get fully damped by the applied thermostat. Supersonic shock waves from collision cascades have been identified in calculations of 100 $\mathrm{keV}$ up to $200 \mathrm{keV}$ recoils in Fe [20]. Note that the oscillations are the same for the two temperatures studied. These oscillations show the influence of slight stress changes on the dislocations, changes that do not affect the defects produced by the collision cascade.

Finally, we show in figure 4 the $z$ distance between dislocations (black line) and the number of vacancies (red line) as a function of time. It is important to point out that the maximum number of displacements in the collision cascade occurs between 2 to 3 ps after the initial PKA and decreases very rapidly. At 10 130 ps the total number of defects remains constant, meaning both the ballistic and thermal spike phases of the collision cascade are over (see figure in Supplemental Material). Therefore, the motion of the dislocations occurs induced by the cascade but equilibrium for the dipole is recovered in a much longer time scale 


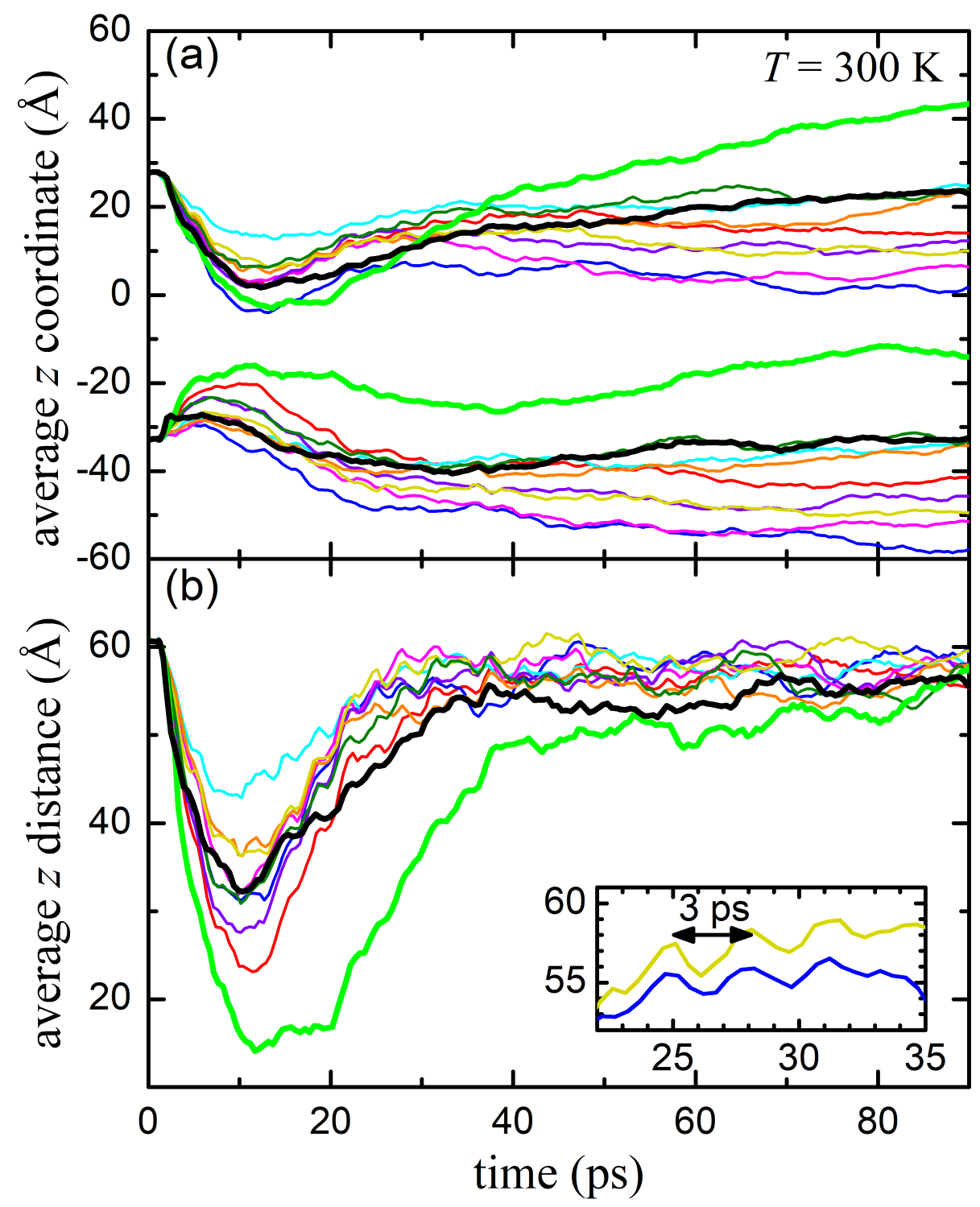

Figure 3: (colour online) (a) Evolution of the average $z$ coordinate of each dislocation and (b) evolution of the $z$ distance between dislocations; after starting a $20 \mathrm{keV}$ cascade close to the $-x$ direction in a bcc Fe with two edge dislocations at $T=300 \mathrm{~K}$. 
than the total time of the collision cascade. In fact, the dislocations start moving

right after the peak of the collision cascade.

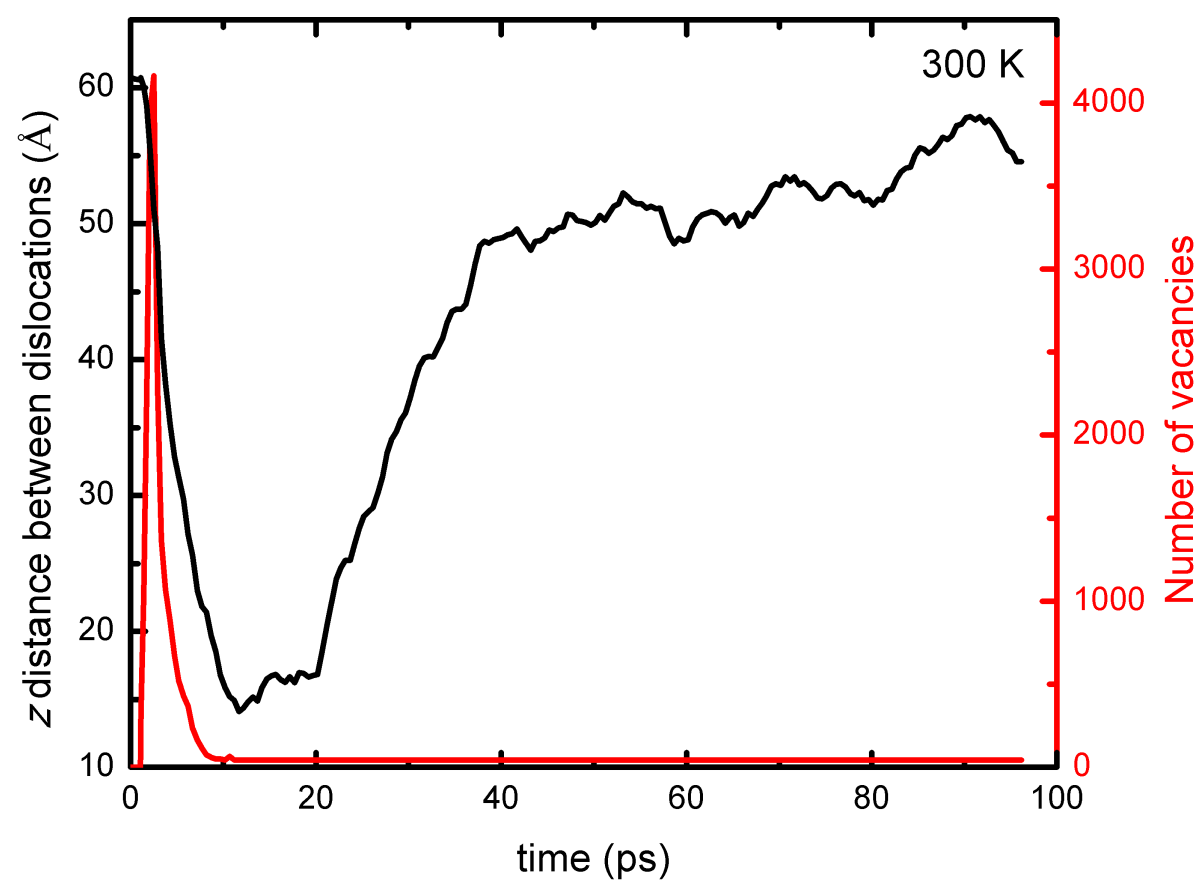

Figure 4: (colour online) $z$ distance between dislocations (black line) and the number of vacancies (red line) as a function of time during, and after, the $20 \mathrm{keV}$ collision cascade at $T=300 \mathrm{~K}$.

Decoupling the contribution to dislocation mobility of the shock wave and temperature gradient produced by the cascade from the production of defects at the dislocations from these simulations is not straightforward. In order to get a better understanding, we have performed simulations where a Gaussian temperature distribution is initiated in the center of the simulation cell, in between the two dislocations. Here no defects are produced. Figure 5 shows the evolution of this temperature profile in time. Atoms identified as defects with the common neighbor analysis in OVITO are represented, with colours corresponding to the $\sigma_{z z}$ component of the atomic level stress. This figure shows

how bending of the dislocation along the $z$ direction, that is, the formation of kinks, and the migration of the dislocation occurs even without the arrival 
of defects to the dislocations. It is important to note that this is different from having a fixed higher temperature in the whole cell, where we saw no motion of the dislocations in the same time scale. Therefore, the motion of these dislocations is driven by the stress created by the temperature gradient. More details about the bending of the dislocations along the $\mathrm{z}$ direction is included in the Supplementary material.

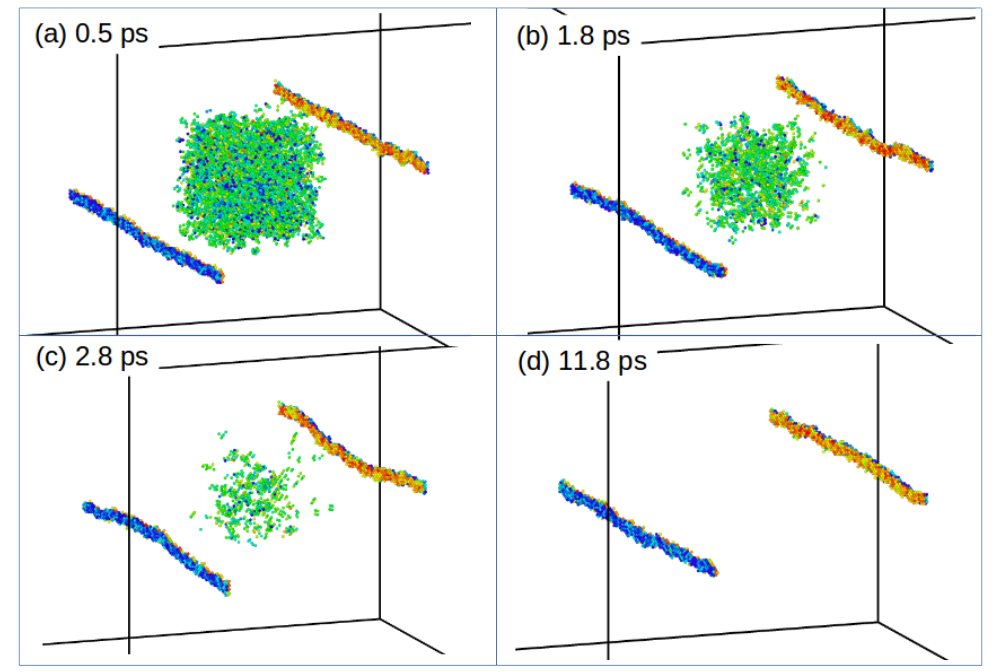

Figure 5: Evolution of a thermal spike started in the middle of the simulation cell. Colours correspond to $\sigma_{z z}$ component of the atomic level stress, with blue representing negative values and red positive ones. Note the bending of the dislocation.

The same bending of the dislocations is observed in the case of collision cascades, as can be seen in figures 7 and 8 below, although sometimes not so clear when defects arrive to the dislocations. Figure 6 shows a cut through the dislocation core, along the glide plane, with the location of the two dislocations in green spheres as identified by the common neighbor analysis and the colors of the rest of the atoms showing the shear atomic level stress $\sigma_{y z}$ component with red positive values and blue negative ones. Top and bottom figures correspond to the two dislocations of the dipole for different times during the collision cascade: 1 ps before the collision cascade ( $\mathrm{a}$ and $\mathrm{d}$ ), and $0.6 \mathrm{ps}$ ( $\mathrm{b}$ and e) and $2.9 \mathrm{ps}$ ( $\mathrm{c}$ and $\mathrm{f}$ ) after the collision cascade. The bending of the dislocations in 
the $\mathrm{z}$ direction is clearly observed and the central panels (b and e) correspond to the moment when the dislocations start to bend. A change in the shear stress $\sigma_{y z}$ distribution close to the dislocations can be observed at this time. In the case of the dislocation on the top figures, defects arrive to the dislocation but not in the dislocation at the bottom. The resulting damage produced in the cascade for this case can be seen in figure 8, on the left panels.
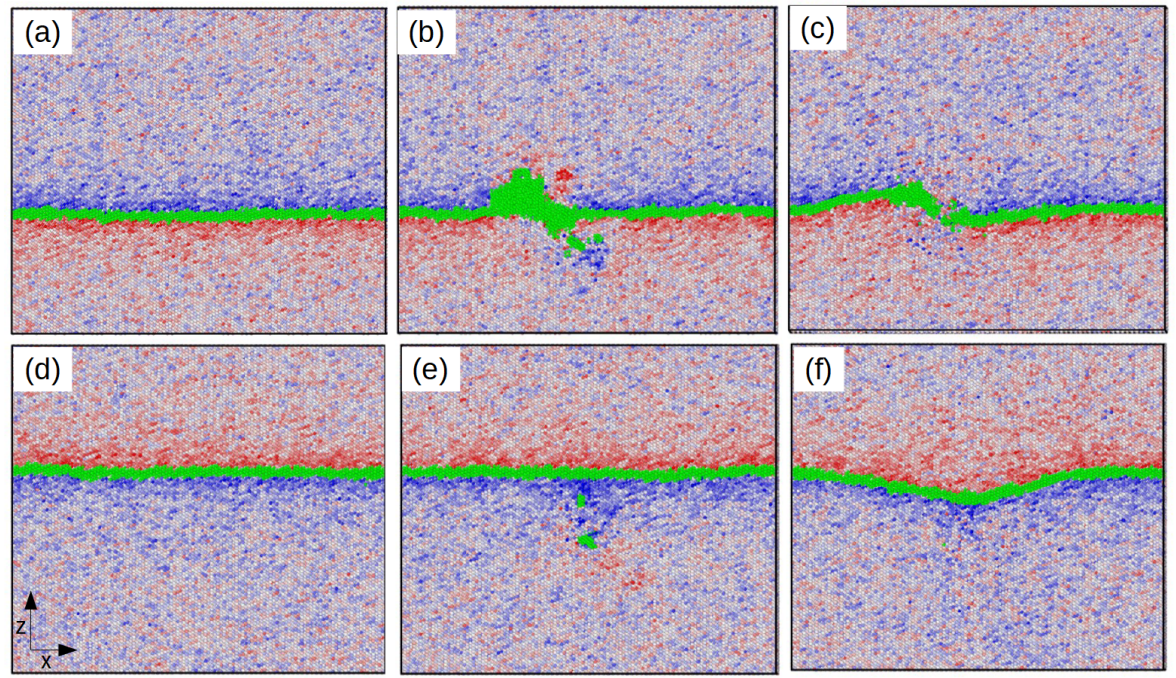

Figure 6: (colour online) Cut through the dislocation core along the glide plane, for different times during the collision cascade and for the two dislocations (top and bottom). Green spheres showing the location of the dislocation line. Colours are the shear $\sigma_{y z}$ component of the atomic level stress.

\subsection{Cascade evolution near the dislocations}

In order to understand the mechanisms and processes occurring during the collision cascade next to the dipole we present in figures 7 and 8 the evolution of different cases at $20 \mathrm{~K}$ and $300 \mathrm{~K}$, respectively. In both figures we show how the system evolved at $1 \mathrm{ps}, 5 \mathrm{ps}$, and $10 \mathrm{ps}$ after starting the energetic recoil. 
The case shown on left side of figure 7 corresponds to the black thick lines

in OVITO, we observe that the interstitial cluster identified by the Wigner-Seitz method it actually corresponds to climb on the dislocation due to the arrival of these defects, as can be seen in figure 9 . 


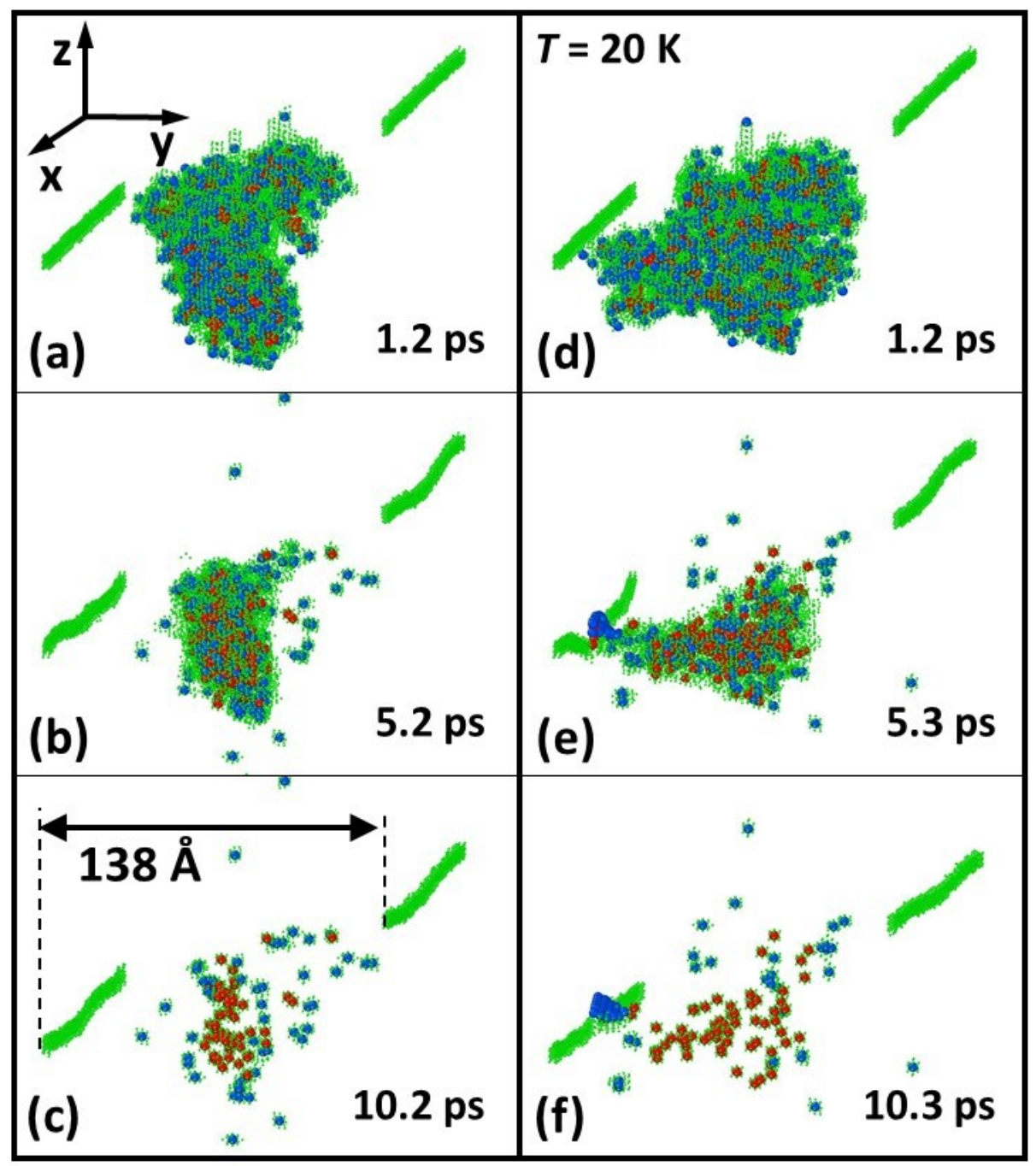

Figure 7: (colour online) Details of the simulation for two selected histories at $T=20 \mathrm{~K}$ for different times after starting the cascade. The history shown on the left (right) hand is represented by black (green) thick lines in figures 2 a) and 2(b). Blue dots represent interstitials, red dots represent vacancies, and green dots show the atoms whose surrounding is different to a b.c.c.; those include the dislocation atoms. 


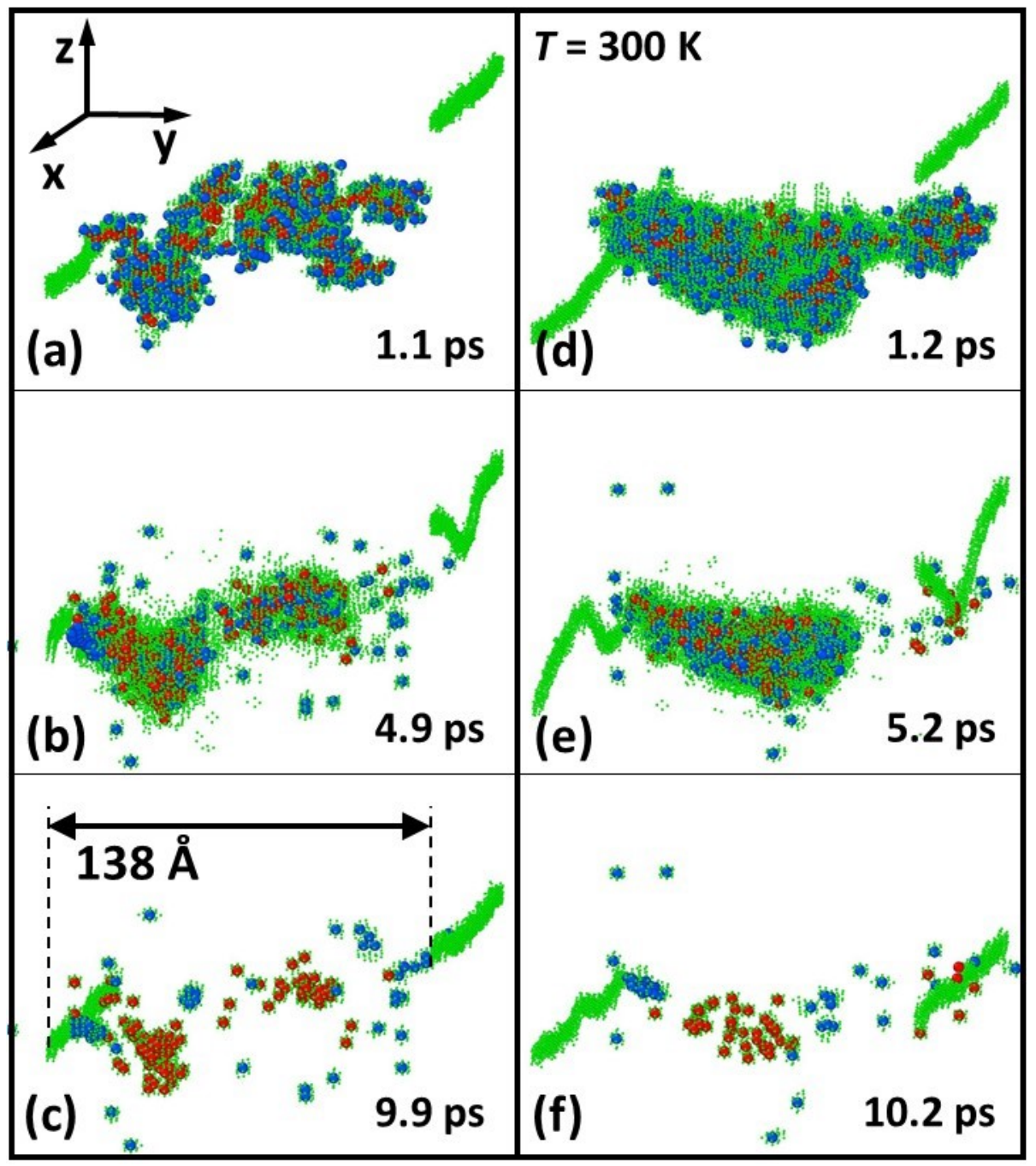

Figure 8: (colour online) Details of the simulation for two selected histories at $T=300 \mathrm{~K}$ for different times after starting the cascade. The history shown on the left (right) is represented by black (green) thick lines in figures 3 (a) and 3(b). Blue dots represent interstitials, red dots represent vacancies and green dots show the atoms whose surrounding is different to a b.c.c.; those include the dislocation atoms. 

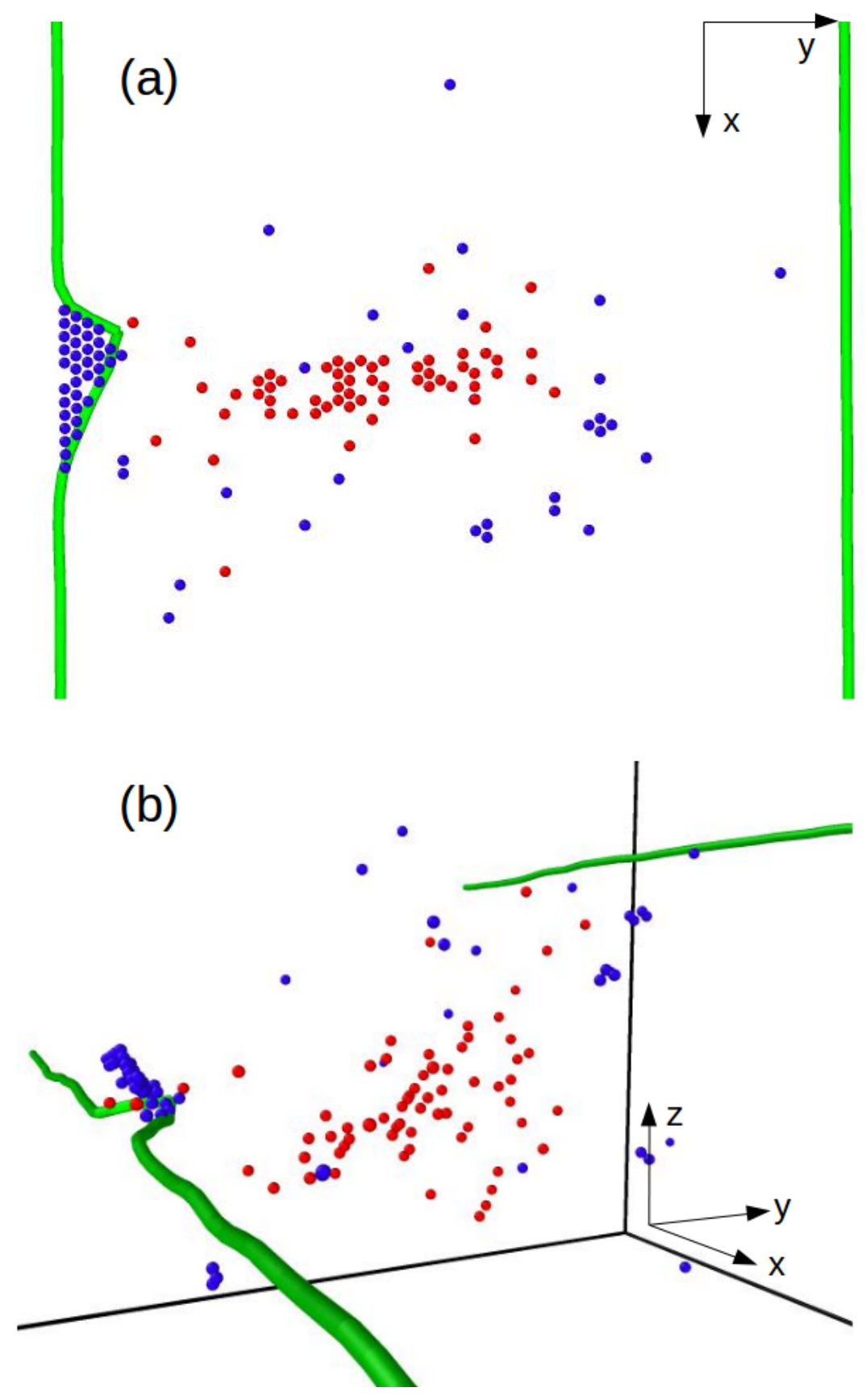

Figure 9: (colour online) Configuration of dislocations after a $20 \mathrm{keV}$ collision cascade at 20 $\mathrm{K}$ from two different perspectives, one a projection along the $z$ axis (a), corresponding to the case shown in figure 7 (f). 
It is clear from these figures, the influence of the dislocations on the shape of the collision cascade: the cascades are elongated, perpendicular to the dislocation lines. This is more clearly observed when representing the shear component of the atomic level stress in a cut through the center of the collision cascade, as shown in figure 10

On the left hand of figure 8 , we can see that the cascade breaks into two subcascades, giving rise to the formation of two clusters of vacancies as well as a cluster of interstitials next to the left dislocation. This cascade corresponds to the black thick lines in figure 3 . Here the two dislocations get close to each

well as figure $8(\mathrm{f})$. In this case, the dislocations also move back to recover the equilibrium distance, although now the time to reach that equilibrium is longer than in the other cases where the dislocations did not approach each other so much (see figure 3(b)). The whole evolution of these cascades are provided as 230 supplemental material in movies 3 and 4 .

\subsection{Analysis of resulting damage: vacancies and self-interstitials}

We have performed a statistical analysis of the defects produced in the different cases: with and without dislocations and two temperatures $(20 \mathrm{~K}$ and $300 \mathrm{~K})$. Although the total number of simulations performed for each condition 

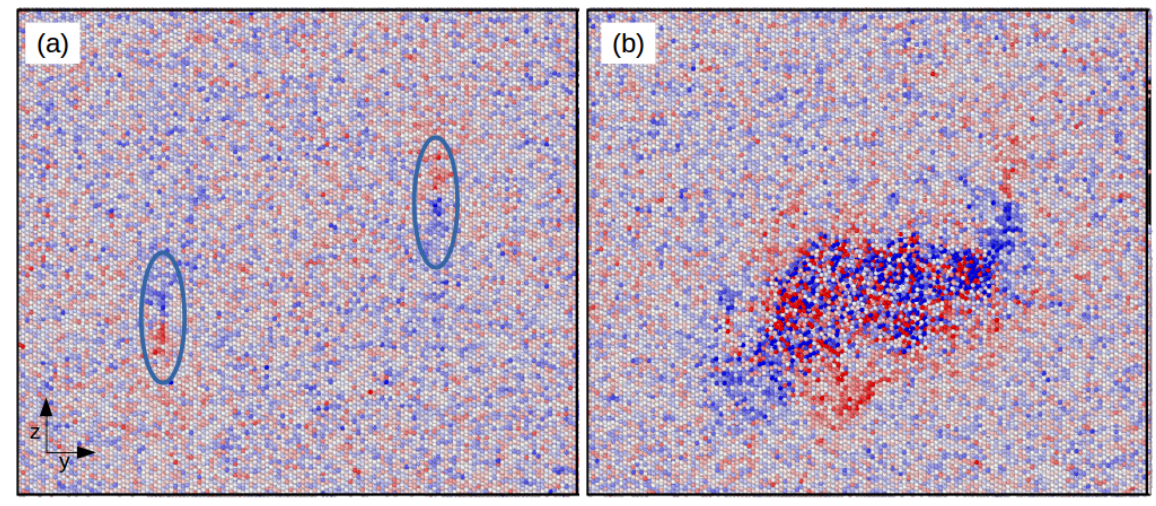

Figure 10: (colour online) Cut through the center of the simulation box, color representing the shear stress $\sigma_{y z}$ component of the atomic level stress, with red positive values and blue negative ones for the initial distribution (a) and the distribution at the peak of the collision cascade (b). The circles indicate the location of the dislocation cores.

is low (10 cases), some conclusions can be extracted from this analysis. From the Wigner-Seitz analysis, we have calculated the size of the different clusters of vacancies and self-interstitials created at the end of the collision cascade. Following the criteria of Björkas et al. 21], for the case of vacancies, the cut-off distance is second nearest neighbors while for self-interstitials is third nearest neighbors to consider two defects as belonging to the same cluster. From this analysis we obtained the percentage of vacancies/self-interstitials in clusters and the largest vacancy/self-interstitial cluster produced. All these values are included in table 1).

When looking at the total number of Frenkel-pairs produced (third column in table 1 no significant differences can be inferred from these results, except for, perhaps a slightly lower number of defects at $300 \mathrm{~K}$ compared to $20 \mathrm{~K}$, independently of the presence or not of the dislocations. When analyzing the percentage of defects in clusters, at first glance it would seem that the percentage of vacancies in clusters decreases when the dislocations are present, while for 


\section{Conclusions}

We have studied the influence of dislocations on the evolution of collision cascades in b.c.c. Fe. An edge dislocation dipole is considered where the effects of the dislocations on the collision cascade is more clearly observed. These simulations show that the recoils induce glide of the dislocations, occurring in the early stages of the collision cascade and related to the shock wave produced by the energetic recoil. The dislocations also influence the morphology of the collision cascade, being mostly elongated and perpendicular to the dislocation 


\begin{tabular}{||ccccccccc||}
\hline$T(\mathrm{~K})$ & Dipole & FP & $\% \mathrm{~V}-\mathrm{C}$ & $\% \mathrm{I}-\mathrm{C}$ & $\left\langle\mathrm{V}_{\max }\right\rangle$ & $\left\langle\mathrm{I}_{\max }\right\rangle$ & $\mathrm{V}_{\max }$ & $\mathrm{I}_{\max }$ \\
\hline \hline 20 & No & $57 \pm 4$ & $41 \pm 3$ & $34 \pm 3$ & $7 \pm 1$ & $5 \pm 1$ & 13 & 9 \\
20 & Yes & $60 \pm 4$ & $27 \pm 3$ & $41 \pm 5$ & $7 \pm 2$ & $10 \pm 3$ & 20 & 35 \\
300 & No & $47 \pm 3$ & $45 \pm 5$ & $52 \pm 3$ & $10 \pm 2$ & $10 \pm 2$ & 26 & 21 \\
300 & Yes & $51 \pm 6$ & $37 \pm 7$ & $58 \pm 7$ & $12 \pm 4$ & $21 \pm 8$ & 38 & 58 \\
\hline
\end{tabular}

Table 1: Analysis of defects for the two temperatures, with and without the dipole. The table includes the average number of Frenkel pairs (FP), the percentage of vacancies in clusters (\% $\mathrm{V}-\mathrm{C}$ ), percentage of self-interstitials in clusters (\% I-C), the average maximum cluster size for vacancies $\left(\left\langle\mathrm{V}_{\max }\right\rangle\right)$ and interstitials $(\langle\mathrm{I} \max \rangle)$ and the maximum vacancy $\left(\mathrm{V}_{\max }\right)$ and interstitial (Imax) cluster obtained.

line instead of almost spherical as in a pristine cell. Dislocation climb is also observed when the collision cascade surrounds the dislocation, leaving behind a vacancy cluster close to the dislocation. In general, we observe that the presence of dislocations results in the formation of larger vacancy clusters.

\section{CREdiT authorship contribution statement}

S. Heredia-Avalos: Conceptualization, Methodology, Software, Validation, Formal analysis, Investigation, Writing - Original Draft, Writing - Review \& Editing, Visualization. C. D. Denton: Conceptualization, Methodology, Software, Validation, Formal analysis, Investigation, Writing - Original Draft, Writing - Review \& Editing, Visualization. J. C. Moreno-Marín: Conceptualization, Methodology, Software, Validation, Formal analysis, Investigation, Writing - Original Draft, Writing - Review \& Editing, Visualization. E. Martinez: Methodology, Writing - Review \& Editing. M. J. Caturla: Conceptualization, Methodology, Software, Validation, Formal analysis, Investigation, Resources, Writing - Review \& Editing, Visualization, Project administration, Funding acquisition. 


\section{Acknowledgements}

This work was partly supported by the Generalitat Valenciana through PROMETEO2017/139. This work has been carried out within the framework of the EUROfusion Consortium and has received funding from the Euratom research and training programme 2014-2018 and 2019-2020 under grant agreement No 633053. The views and opinions expressed herein do not necessarily reflect those of the European Commission. EM gratefully acknowledges support from the U.S. DOE, Office of Science, Office of Fusion Energy Sciences, and Office of Advanced Scientific Computing Research through the Scientific Discovery through Advanced Computing (SciDAC) project on Plasma-Surface Interactions (award no. DE-SC0008875).

\section{Data availability}

The raw/processed data required to reproduce these findings cannot be shared at this time due to legal or ethical reasons.

\section{References}

[1] T. A. Khraishi, H. M. Zbib, T. D. de la Rubia, M. Victoria, Localized deformation and hardening in irradiated metals: Three-dimensional discrete dislocation dynamics simulations, Metallurgical and Materials Transactions B 33 (2) (2002) 285-296. doi:10.1007/s11663-002-0012-7.

URL https://doi.org/10.1007/s11663-002-0012-7

[2] S. J. Zinkle, Microstructures and mechanical properties of irradiated metals and alloys, in: V. Ghetta, D. Gorse, D. Mazière, V. Pontikis (Eds.), Materials Issues for Generation IV Systems, Springer Netherlands, Dordrecht, 2008, pp. 227-244.

[3] G. Pintsuk, E. Diegele, S. Dudarev, M. Gorley, J. Henry, J. Reiser, M. Rieth, European materials development: Results and perspective, Fusion Engineering and Designdoi:10.1016/j.fusengdes.2019.02.063. 
q [4] D. Terentyev, Y. Osetsky, D. Bacon, Competing processes in reac-

n tions between an edge dislocation and dislocation loops in a bodycentred cubic metal, Scripta Materialia 62 (9) (2010) 697 - 700. doi:https://doi.org/10.1016/j.scriptamat.2010.01.034. URL http://www.sciencedirect.com/science/article/pii/ S1359646210000357

¿ [5] D. Bacon, Y. Osetsky, D. Rodney, Chapter 88 dislocation obstacle interactions at the atomic level, Vol. 15 of Dislocations in Solids, Elsevier, 2009, pp. 1 - 90. doi:https://doi.org/10.1016/S1572-4859(09)01501-0

[6] S. Hafez, M. Fivel, J. Fikar, R. Schublin, Dislocationvoid interaction in fe: A comparison between molecular dynamics and dislocation dynamics, 335 Journal of Nuclear Materials 386-388 (2009) 102-105. doi:10.1016/j. jnucmat.2008.12.069.

[ [7] R. E. Voskoboinikov, Interaction of collision cascades with an isolated edge dislocation in aluminium, Nuclear Instruments and Methods in Physics Research Section B: Beam Interactions with Materials and Atoms 303 (2013) 125 - 128, proceedings of the 11th Computer Simulation of Radiation Effects in Solids (COSIRES) Conference Santa Fe, New Mexico, USA, July 24-29, 2012. doi :https://doi.org/10.1016/j.nimb.2012.10.022. URL http://www.sciencedirect.com/science/article/pii/ S0168583X12006593

[8] R. E. Voskoboinikov, Md simulations of collision cascades in the vicinity of a screw dislocation in aluminium, Nuclear Instruments and Methods in Physics Research Section B: Beam Interactions with Materials and Atoms 303 (2013) 104 - 107, proceedings of the 11th Computer Simulation of Radiation Effects in Solids (COSIRES) Conference Santa Fe, New Mexico, USA, July 24-29, 2012. doi:https://doi.org/10.1016/j.nimb.2013.01.021. 
URL http://www.sciencedirect.com/science/article/pii/ S0168583X13001468

[9] B. Q. Fu, S. P. Fitzgerald, Q. Hou, J. Wang, M. Li, Effect of collision cascades on dislocations in tungsten: A molecular dynamics study, $\mathrm{Nu}-$ clear Instruments and Methods in Physics Research Section B: Beam Interactions with Materials and Atoms 393 (2017) 169-173, 13th International Conference on Computer Simulation of Radiation Effects in Solids (COSIRES), Loughborough Univ, Loughborough, ENGLAND, JUN 19-24, 2016. doi:\{10.1016/j.nimb.2016.10.028\}.

[10] H. Wang, J.-T. Tian, W. Zhou, X.-F. Chen, B. Bai, J.-M. Xue, Collision cascades interact with an edge dislocation in bcc fe: a molecular dynamics study, RSC Adv. 8 (2018) 14017-14024. doi:10.1039/C8RA00141C URL http://dx.doi.org/10.1039/C8RA00141C

[11] Z. Lu, L. Xu, T. Chen, L. Tan, H. Xu, Interactions between displace365 ment cascade and dislocation and their influences on peierls stress in fe-20cr-25ni alloys, Computational Materials Science 160 (2019) 279 - 286. doi:https://doi.org/10.1016/j.commatsci.2018.12.018. URL http://wwW.sciencedirect.com/science/article/pii/ S0927025618307973

[12] D. H. Thompson, E. Tarleton, S. G. Roberts, S. P. Fitzgerald, a Interstitial-mediated dislocation climb and the weakening of particlereinforced alloys under irradiation, Phys. Rev. Materials 2 (2018) 080601. doi:10.1103/PhysRevMaterials.2.080601.

I URL https://link.aps.org/doi/10.1103/PhysRevMaterials.2. 375 080601

[13] S. Plimpton, Fast parallel algorithms for short-range molecular dynamics J. Comp. Phys. 117 (1) (1995) 1-19. doi:10.1006/jcph.1995.1039.

[ URL https://www.sciencedirect.com/science/article/pii/ S002199918571039X 
[17] A. Stukowski, Visualization and analysis of atomistic simulation data with OVITO-the open visualization tool, Modelling and Simulation in Materials

(1)

[18] J. D. Honeycutt, H. C. Andersen, Molecular dynamics study of melting and freezing of small lennard-jones clusters, The Journal of Physical

[19] A. Stukowski, V. V. Bulatov, A. Arsenlis, Automated identification and indexing of dislocations in crystal interfaces, Modelling and Simulation in $0965-0393 / 20 / 8 / 085007$

405

[20] Q. Peng, F. Meng, Y. Yang, C. Lu, H. Deng, L. Wang, S. De, F. Gao, Shockwave generates $<100>$ dislocation loops in bcc iron, Nature Com- 
munications 9 (1) (2018) 4880. doi:10.1038/s41467-018-07102-3.

URL https://doi .org/10.1038/s41467-018-07102-3

[21] C. Björkas, K. Nordlund, Comparative study of cascade damage in fe simulated with recent potentials, Nuclear Instruments and Methods in Physics Research Section B: Beam Interactions with Materials and Atoms 259 (2) (2007) 853 - 860. doi:https://doi.org/10.1016/j.nimb.2007.03.076

口

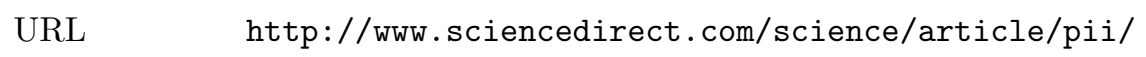

\section{Supplementary material}

The files movie1.avi and movie2.avi show collision cascades at $20 \mathrm{~K}$ depicted on figure 7, whereas the files movie3.avi and movie4.avi show the collision cascades at $300 \mathrm{~K}$ depicted on figure 8 . captions for additional information. 

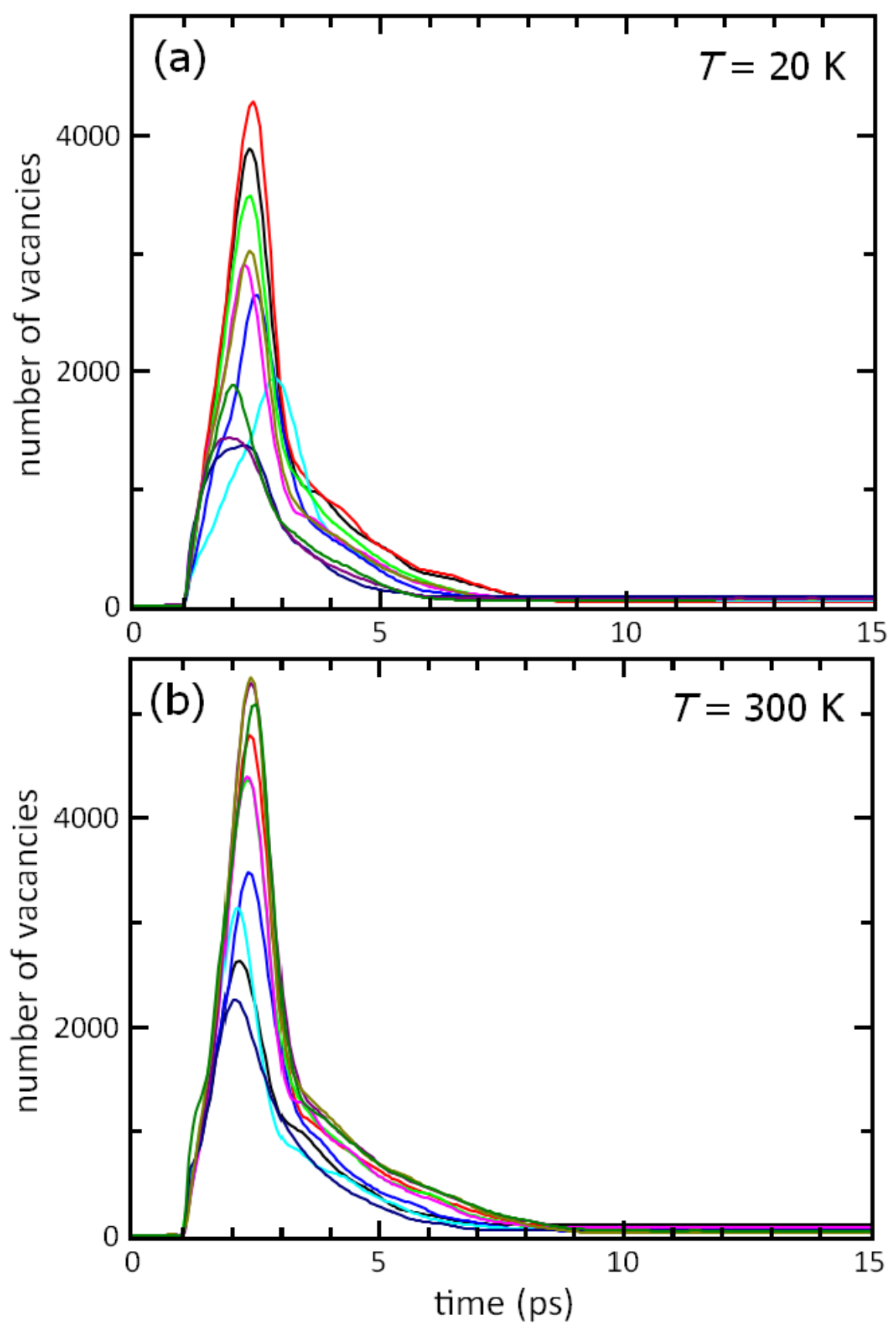

Figure 11: Evolution of the number of vacancies on bcc Fe with two dislocations after the cascade is started for (a) $T=20 \mathrm{~K}$ and (b) $T=300 \mathrm{~K}$. Notice that after the cascade phase, the number of vacancies reaches an equilibrium value at $10 \mathrm{ps}$. 

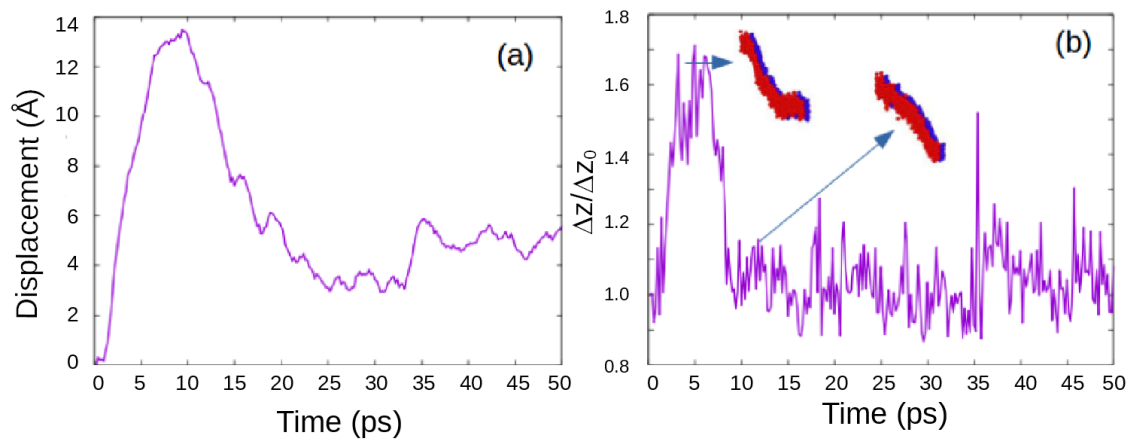

Figure 12: (a) Displacement of a dislocation during a thermal spike; we have analyzed the bending of the dislocation along the $z$ direction by calculating the maximum and minimum values of $z$ in the dislocation $(\Delta z)$ and dividing this value with respect to the initial value which would be a measure of the width of this dislocation $\left(\Delta z_{0}\right)$. (b) Ratio of $\Delta z / \Delta z_{0}$ (b); the largest bending along the $\mathrm{z}$ direction is observed at the beginning of the temperature spike. 


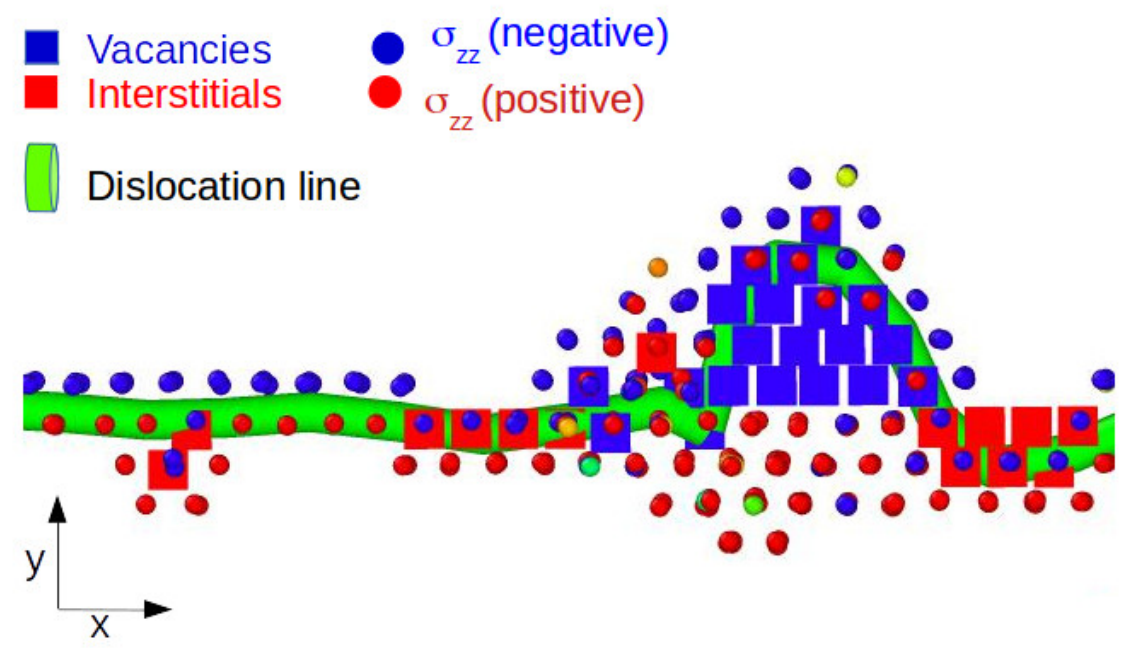

Figure 13: An example of the location of vacancies and self-interstitials around a dislocation in relation to the stress distribution. 